\title{
Radio-frequency transparent demodulation for broadband hybrid wireless-optical links
}

Zibar, Darko; Sambaraju, Rakesh; Alemany, Ruben; Caballero Jambrina, Antonio; Herrera, Javier; Tafur Monroy, Idelfonso

Published in:

I E E E Photonics Technology Letters

Link to article, DOI:

10.1109/LPT.2010.2045752

Publication date:

2010

Document Version

Publisher's PDF, also known as Version of record

Link back to DTU Orbit

Citation (APA):

Zibar, D., Sambaraju, R., Alemany, R., Caballero Jambrina, A., Herrera, J., \& Tafur Monroy, I. (2010). Radiofrequency transparent demodulation for broadband hybrid wireless-optical links. I E E E Photonics Technology Letters, 22(11), 784-786. https://doi.org/10.1109/LPT.2010.2045752

\section{General rights}

Copyright and moral rights for the publications made accessible in the public portal are retained by the authors and/or other copyright owners and it is a condition of accessing publications that users recognise and abide by the legal requirements associated with these rights.

- Users may download and print one copy of any publication from the public portal for the purpose of private study or research.

- You may not further distribute the material or use it for any profit-making activity or commercial gain

- You may freely distribute the URL identifying the publication in the public portal 


\title{
Radio-Frequency Transparent Demodulation for Broadband Hybrid Wireless-Optical Links
}

\author{
Darko Zibar, Rakesh Sambaraju, Ruben Alemany, Antonio Caballero, Javier Herrera, and Idelfonso T. Monroy
}

\begin{abstract}
A novel demodulation technique which is transparent to radio-frequency (RF) carrier frequency is presented and experimentally demonstrated for multigigabit wireless signals. The presented demodulation technique employs optical single-sideband filtering, coherent detection, and baseband digital signal processing. Multigigabit wireless signal demodulation of 1.25-Gbaud quadrature phase-shift-keying modulated data at 40- and 35-GHz RF carrier frequency is experimentally demonstrated using the proposed demodulation scheme.
\end{abstract}

Index Terms-Coherent communication, digital receivers, digital signal processing, microwave photonics, modulation.

\section{INTRODUCTION}

$\mathbf{T}$ HE increase in the data capacity demand is pushing the development of multigigabit wireless systems. Currently, there is much research effort underway in order to develop multigigabit wireless systems addressing applications like local area network (LAN) bridging, interbuilding communications, mobile backhaul, etc. [1]-[3]. Several frequency bands in the millimeter-wave frequency regime $(60,70 / 80$, $>100 \mathrm{GHz}$, etc.) have a few gigahertz of available bandwidth, which could potentially enable gigabit wireless transmissions. However, in order to achieve multigigabit capacity in the limited available millimeter-wave bandwidth, spectrally efficient modulation formats like quadrature phase-shift-keying (QPSK) and $M$-quadrature amplitude modulation (QAM) are required. Radio-frequency (RF)-over-fiber technologies [3] provide a good solution for generation and transport of wireless signals. Wireless signal generation with the bit rate up to $10 \mathrm{~Gb} / \mathrm{s}$, has been generated using on-off keying and spectral efficient QAM modulation [2], [5]. However, the detection of these vector modulated multigigabit signals using conventional electrical methods becomes complicated when the bit rate increases and RF carrier frequency approaches millimeter-wave frequencies. Recently, several photonic techniques for demodulation of $M$-QAM signals have been proposed ([4], [5] and

Manuscript received January 06, 2010; revised February 16, 2010; accepted March 07, 2010. Date of publication March 18, 2010; date of current version May 07, 2010. This work has been supported by the European Commission FP7 under Network of Excellence project EUROFOS (22402).

D. Zibar, A. Caballero, and I. T. Monroy are with DTU Fotonik, Department of Photonics Engineering, Technical University of Denmark, Kgs. Lyngby, DK-2800, Denmark (e-mail: dazi@fotonik.dtu.dk; dz@com.dtu.dk; acaj@fotonik.dtu.dk; idtm@ fotonik.dtu.dk).

R. Sambaraju, R. Alemany, and J. Herrera are with Valencia Nanophotonics Technology Center, Universidad Politécnica de Valencia, 46022 Valencia, Spain (e-mail: rsambaraju@ntc.upv.es; ralemany@ntc.upv.es; jherrera@ntc.upv.es).

Color versions of one or more of the figures in this letter are available online at http://ieeexplore.ieee.org.

Digital Object Identifier 10.1109/LPT.2010.2045752 references therein), but they still require relatively complex high-bandwidth analog phase-locked-loop and RF components. Additionally, digital coherent receiver structures have also been proposed for RF-over-fiber links [6], [7]. Although, the demonstrated digital coherent techniques do not require electronic or optical phase-locked loops, they still require electronics such as analog-to-digital (A/D) converters or signal sources at high RF carrier frequencies [8]. In this letter, we consider the optical link between the remote antenna unit and the central station for the delivery of high RF carrier frequency broadband wireless signals. We present a novel technique for demodulation of high-frequency multigigabit RF signals using coherent detection and baseband digital signal processing. The advantage of the proposed technique is that it is transparent to RF carrier frequency and only requires $\mathrm{A} / \mathrm{D}$ converters at the baseband/data rate frequency. In order to demonstrate the RF frequency transparency, demodulation of $2.5-\mathrm{Gb} / \mathrm{s}$ QPSK modulated data signal, at 40- and 35-GHz RF carrier frequency, is experimentally demonstrated. To the best of our knowledge, this is the first experimental demonstration where a combination of optical single-sideband filtering, coherent detection, and baseband digital signal processing is employed for fiber transport and subsequent demodulation of high RF carrier frequency, phase-shift-keyed modulated wireless signals. It is this unique combination that makes our proposed technique transparent to the RF carrier frequency as well as broadband.

\section{EXPERIMENTAL SETUP AND DigITAL COHERENT DEMODULATION}

The schematic of the experimental setup is shown in Fig. 1. The system incorporates fiber to attain a simplified remote antenna base station and thereby locate all the signal processing parts at the central station. The idea is to keep the remote antenna station as simple as possible [3]. In order to emulate the received RF signal at the remote antenna station, a pattern generator is used to generate in-phase and quadrature components of the 1.25-Gbaud QPSK data signal which are then up-converted to an RF carrier frequency of $f_{\mathrm{RF}}=40 \mathrm{GHz}$ by a vector signal generator. The electrical power of the QPSK modulated RF signal was set to $+2 \mathrm{dBm}$. An optical carrier at the wavelength of $\lambda_{1}=1554.68 \mathrm{~nm}$ generated from an external cavity tunable laser (TLS) was amplitude modulated with the 1.25-Gbaud QPSK modulated data signal at the $40-\mathrm{GHz}$ RF carrier frequency using a Mach-Zehnder modulator (MZM) biased at the quadrature point. The output of the MZM consists of the optical carrier at $\lambda_{1}=1554.68 \mathrm{~nm}$, and two sidebands, at $\lambda_{1}-\lambda_{\mathrm{RF}}=1554.36 \mathrm{~nm}$ and $\lambda_{1}+\lambda_{\mathrm{RF}}=1555.0 \mathrm{~nm}$, each modulated with 1.25-Gbaud QPSK data and separated by 


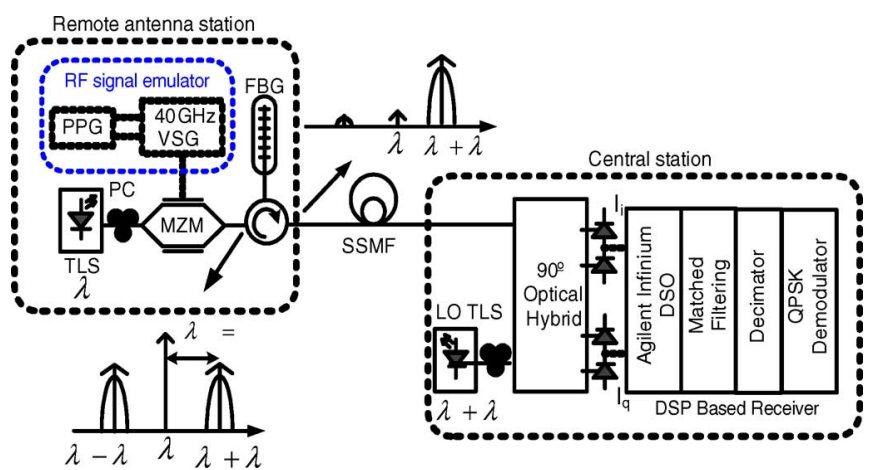

Fig. 1. General outline for the experimental setup for demonstration of RF transparent demodulation of multigigabit RF-over-fiber link. PPG: pulse pattern generator; VSG: vector signal generator; FBG: fiber Bragg grating; MZM: Mach-Zehnder modulator; PC: polarization controller; TLS: tunable laser source; LO: local oscillator; $\lambda_{1}$ : wavelength of the TLS; $\lambda_{1}+\lambda_{\mathrm{RF}}$ : wavelength of the LO TLS; $f_{\mathrm{RF}}$ : RF carrier frequency of the signal from VSG.

$40 \mathrm{GHz} / 0.32 \mathrm{~nm}$ from $\lambda_{1}$ (see Fig. 1). The main idea behind our approach is now to filter out one of the sidebands of the modulated optical carrier $\left(\lambda_{1}+\lambda_{\mathrm{RF}}\right.$ or $\left.\lambda_{1}-\lambda_{\mathrm{RF}}\right)$, and in this way only transmit baseband data making this scheme RF carrier frequency independent, and also dispersion tolerant since only the baseband data is transmitted. This is illustrated in Fig. 1. To filter out the sideband at $\lambda_{1}+\lambda_{\mathrm{RF}}=1555.0 \mathrm{~nm}$, a fiber Bragg grating (FBG) centered at $\lambda_{1}+\lambda_{\mathrm{RF}}=1555.0 \mathrm{~nm}$ with an optical bandwidth of $25 \mathrm{GHz}$ is used in combination with an optical circulator. The reflected signal from the FBG at the wavelength $\lambda_{1}+\lambda_{\mathrm{RF}}=1555.0 \mathrm{~nm}$ which is then sent to the receiver contains purely $2.5-\mathrm{Gb} / \mathrm{s}$ QPSK data modulation. The optical data signal at $\lambda_{1}+\lambda_{\mathrm{RF}}=1555.0 \mathrm{~nm}$, carrying $2.5 \mathrm{~Gb} / \mathrm{s}$ of QPSK data, is then transmitted over a $26-\mathrm{km}$ standard single-mode fiber (SMF) prior to coherent detection, which only requires baseband signal processing at twice the data rate. At the receiver, an optical local oscillator (LO) signal, generated from another tunable external cavity laser ( $\sim 100-\mathrm{kHz}$ linewidth), and with the wavelength approximately set to $\lambda_{1}+\lambda_{\mathrm{RF}}=1550.0 \mathrm{~nm}$ is mixed with the filtered sideband carrying $2.5-\mathrm{Gb} / \mathrm{s}$ QPSK data in the $90^{\circ}$ optical hybrid. We have a polarization controller after the LO laser output so that the polarization of the LO optical signal is manually aligned to maximize the power input to the optical hybrid. In practice, a polarization diversity scheme or polarization tracking could be employed. The in-phase $I_{i}$ and quadrature $I_{q}$ optical components are detected using two pairs of balanced photodiodes (BW 7.5 GHz), which were inbuilt in the optical $90^{\circ}$ optical hybrid. The photodetected signals were then digitized using a high bandwidth real-time oscilloscope. The digitized signals $I_{i}[k]$ and $I_{q}[k]$, where $k$ is an integer, are later used for offline digital signal processing consisting of matched filtering, decimation, and a QPSK demodulation module. The matched filter is a finite impulse response (FIR) raised cosine filter and is used to maximize the signal-to-noise ratio of the detected signal. The decimator down-samples the digitized signals $I_{i}[k]$ and $I_{q}[k]$ to one sample per bit prior to QPSK demodulation block. The QPSK demodulation block is a decision directed feedback structure which removes phase and frequency offset between the transmitter and LO laser and, thereby, performs QPSK data signal demodulation [9]. The fre-

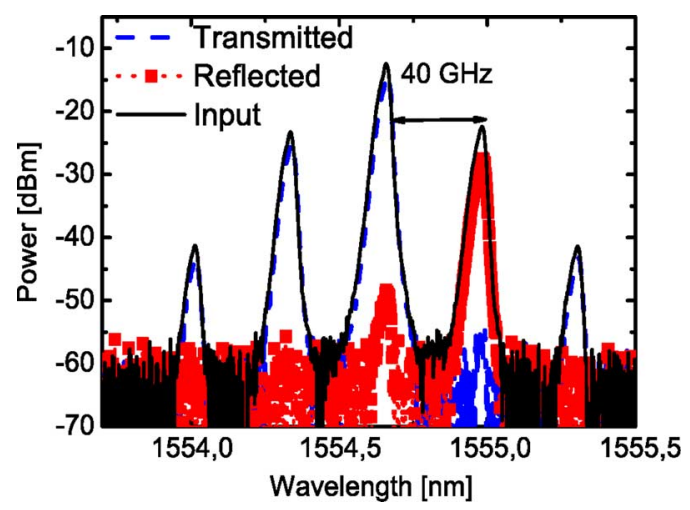

Fig. 2. Optical spectrum of the input signal to the FBG, and reflected and transmitted optical signal through the FBG.

quency offset between the transmitter laser and the LO may approach up to $500 \mathrm{MHz}$, making its removal quite challenging by using conventional digital estimation methods like Viterbi \& Viterbi, for which frequency offset values up to approximately $10 \%$ of the baud rate can be tolerated [9]. Therefore, we use a feedback structure employing a digital phasor-locked loop such that frequency offset values up to $500 \mathrm{MHz}$ can be removed by optimizing the loop bandwidth and still keeping the loop stable. Since the power of the received RF signal may in some cases be very low, it would result in very low sideband powers as well. If no optical single sideband filtering is used, then the gain of the optical amplifiers would be distributed over the entire optical signal, including the carrier and the other sideband which is not desired. The optical single-sideband filtering can be placed either at the antenna or central office all depending on the transmissions scenario.

\section{EXPERIMENTAL RESULTS}

In Fig. 2, optical spectra of the input signal at the FBG, as well as the optical spectra of the transmitted and the reflected signal from the FBG, are shown. It is the reflected signal at $\lambda_{1}+$ $\lambda_{\mathrm{RF}}=1550.0 \mathrm{~nm}$ containing only baseband $2.5-\mathrm{Gb} / \mathrm{s}$ QPSK data modulation that is sent to the transmission span of $26 \mathrm{~km}$ and subsequent signal demodulation as mentioned earlier. It is observed from Fig. 2 that the FBG suppresses the optical carrier at $\lambda_{1}$ and the lower sideband at $\lambda_{1}-\lambda_{\mathrm{RF}}$ by more than $30 \mathrm{~dB}$, allowing for pure baseband transmission.

In Fig. 3(a), the frequency spectrum of the sampled photocurrent $I_{i}[k]$ is shown. It is observed that the signal is in baseband and is symmetric around zero since the frequency offset between the transmitter and LO laser has been removed by the DSP. The frequency spectra has nulls at $1.25 \mathrm{GHz}$ as expected. In Fig. 3(b), the constellation diagram of the demodulated 2.5-Gb/s QPSK signal is shown for the RF power of $2 \mathrm{dBm}$. The constellation points look clear and well separated indicating that the digital signal-processing-based receiver can be successfully used for QPSK signal demodulation. Next, in order to test the demodulation scheme, we investigate the system performance as a function of optical signal-to-noise ratio (OSNR). Fig. 4(a) shows the bit-error-ratio (BER) curves plotted as a function of the OSNR measured in 0.1-nm resolution bandwidth. To begin with, we focus on the data signal at 


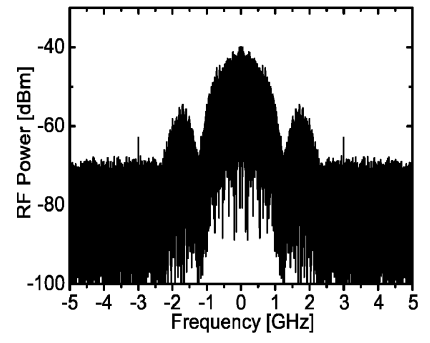

(a)

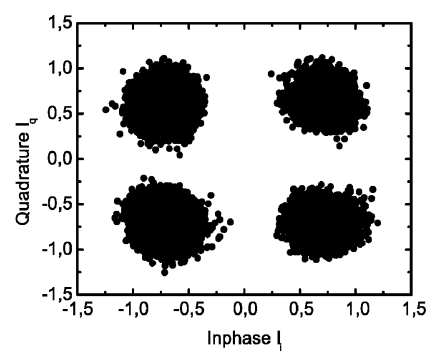

(b)
Fig. 3. (a) Frequency spectra of the sampled photocurrent, $I_{i}[k]$ at the output from one of the arms of the $90^{\circ}$ optical hybrid. (b) Constellation diagram of the demodulated 2.5-Gb/s QPSK data signal.

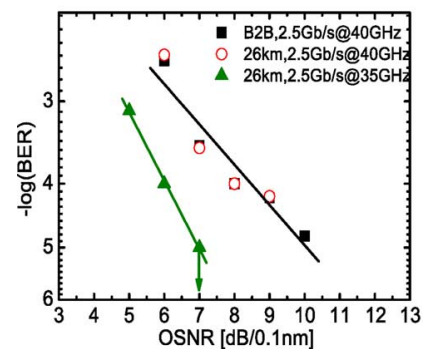

(a)

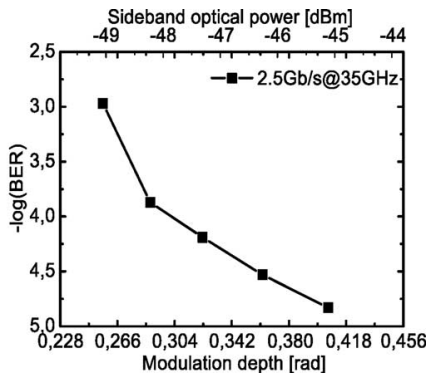

(b)
Fig. 4. (a) BER plotted as a function of OSNR. The RF power is maintained constant at $2 \mathrm{dBm}$, which converts to a modulation depth of $0.51 \mathrm{rad} . V_{\pi}$ of the modulator is $4.9 \mathrm{~V}$. (b) BER plotted as a function of the modulation depth.

40-GHz RF carrier frequency. First, the BER was measured in a back-to-back scenario, and then a 26-km fiber transmission was performed. Fig. 4(a) shows that for the back-to-back scenario, successful signal demodulation is achieved with the receiver sensitivity of $8 \mathrm{~dB}$ in order to obtain the BER of $10^{-4}$. Additionally, it can be observed from Fig. 4(a), that the 26-km fiber transmission did not induce any penalty for the demodulation of a $2.5-\mathrm{Gb} / \mathrm{s}$ QPSK signal at 40-GHz RF carrier, proving the dispersion tolerance of the proposed scheme. In order to demonstrate the RF transparency of the demodulation scheme, the RF carrier frequency was changed to $35 \mathrm{GHz}$ while still keeping the 1.25-Gbaud QPSK data signal modulation. Changing the RF carrier frequency from 40 to $35 \mathrm{GHz}$ has moved the sideband center wavelength, so in order to be able to filter the sideband properly, the transmitter laser was detuned to maximize the filtered optical power. In Fig. 4(a), the BER as a function of OSNR is plotted when the RF carrier frequency is $35 \mathrm{GHz}$. The error-free (zero errors counted) signal demodulation is achieved when the OSNR is $7 \mathrm{~dB}$, which is indicated by an arrow in Fig. 4(a). We define an error-free operation when we observe no errors for the considered 100.000 bits. An improvement in the BER is observed compared to the case when the RF carrier frequency was $40 \mathrm{GHz}$, which is due to the increased frequency response of the MZM at $35 \mathrm{GHz}$ compared to $40 \mathrm{GHz}$. From the specifications of the used MZM provided by the manufacturer, a difference of $2 \mathrm{~dB}$ in $S_{21}$ is noted and this difference is reflected in the measured OSNR penalty (for BER of $10^{-4}$ ) when moving from 35 to $40 \mathrm{GHz}$.

In order to analyze the effect of the RF power on the performance of the demodulation scheme, the BER was measured as a function of the modulation depth without any additional optical noise and plotted in Fig. 4(b). The modulation depth was varied from 0.25 to $0.40 \mathrm{rad}$, and the corresponding optical sideband power was also measured and is shown as well in Fig. 4(b). Fig. 4(b) shows that the BER well below $10^{-4}$ is obtainable. Also, for relatively low sideband optical power of $-48 \mathrm{dBm}$, a BER of approximately $10^{-4}$ is achievable. This proves the high sensitivity of the proposed demodulation scheme. In general, decreasing the modulation depth results in decreasing the signal-to-noise-ratio of the received signal and this translates into increased BER, as shown in Fig. 4(b).

\section{CONCLUSION}

We demonstrate by a proof-of-principle a solution/system for transport of wireless signals from the antenna base station to the central office which is transparent to the RF carrier frequency and only needs electronics at twofold of the wireless signal data rate. The proposed technique is based on optical single-sideband filtering, coherent detection, and baseband digital signal processing. A successful experimental demodulation of 2.5-Gb/s QPSK data signal at 40- and 35-GHz RF carrier frequency is successfully demonstrated using the same receiver structure thereby demonstrating the RF transparency of the scheme.

\section{REFERENCES}

[1] V. Dyadyuk, J. D. Bunton, J. Pathikulangara, R. Kendall, O. Sevimli, L. Stokes, and D. A. Abbott, "A Multigigabit millimeter-wave communication cystem with improved spectral efficiency," IEEE Microw. Theory Tech., vol. 55, no. 12, pp. 2813-2820, Dec. 2007.

[2] A. Hirata, M. Harada, and T. Nagatsuma, " $120-\mathrm{GHz}$ wireless link using photonic techniques for generation, modulation, and emission of millimeter-wave signals," J. Lightw. Technol., vol. 21, no. 10, pp. 2145-2153, Oct. 2003.

[3] J. Capmany and D. Novak, Nature Photon., vol. 1, pp. 319-330, 2007.

[4] G. K. Gopalakrishnan, R. P. Moeller, M. M. Howerton, W. K. Burns, K. J. Williams, and R. D. Esman, "A low-loss downconverting analog fiber-optic link," IEEE Microw. Theory Tech., vol. 43, no. 9, pp. 2318-2323, Sep. 1995.

[5] R. Sambaraju, J. Palaci, R. Alemany, V. Polo, and J. L. Corral, "Photonic vector demodulation of $2.5 \mathrm{Gbit} / \mathrm{s}$ QAM modulated wireless signals," in Proc. Int. Topical Meeting Microwave Photonics 2008, 2008, pp. $117-120$.

[6] T. R. Clark and M. L. Dennis, "Coherent optical phase modulation link," IEEE Photon. Technol. Lett., vol. 19, no. 16, pp. 1206-1208, Aug. $15,2007$.

[7] D. Zibar, X. Yu, C. Peucheret, P. Jeppesen, and I. T. Monroy, "Digital coherent receiver for phase modulated radio-over-fibre optical links," IEEE Photon. Technol. Lett., vol. 21, no. 3, pp. 155-157, Feb. 1, 2009.

[8] A. Caballero, D. Zibar, and I. T. Monroy, "Digital coherent detection of multi-gigabit 16-QAM signals at $40 \mathrm{GHz}$ carrier frequency using photonic downconversion," in Proc. Eur. Conf. Optical Communications, 2009, pp. 58-59, Paper PD3.3.

[9] H. Meyr, M. Moeneclaey, and S. A. Fechtel, Digital Communication Receivers. Hoboken, NJ: Wiley, 1998. 